\title{
PLAGAS EN CACAOTALES, MUNICIPIO DE SIUNA, 2011
}

\author{
Norlan Alfonso López Rizo ${ }^{[1]}$ \\ Elder Leonel Flores Soza ${ }^{[2]}$ \\ Jamil Castillo Martínez ${ }^{[3]}$ \\ Oscar Montalván Castellón ${ }^{[4]}$
}

\section{Resumen}

Esta investigación se realizó en quince unidades productivas de cacao, distribuidas en cinco comunidades de Siuna; se evaluó la prevalencia de plagas y el grado de afectación en frutos, ramas productivas y flores. Para la recolección de datos se utilizó la técnica de muestreo por transectas sistemáticas distribuidas en $12 \%$ por plantación en rangos altitudinales menores a 200, de 200 a 300 y mayores a $300(\mathrm{msnm})$, la información se recolectó mediante la observación directa, se cuantificaron las pérdidas económicas causadas. La prevalencia de plagas es de $74 \%$ y la ausencia de $26 \%$ en alturas superiores a $300(\mathrm{msnm})$. La tanda sobresale en las 15 plantaciones, con 50\% en rangos menores de $200 \mathrm{msnm}, 38 \%$ de 200 a 300 y $64 \%$ mayores de $300 \mathrm{msnm}$, la ardilla con $34 \%, 39 \%$ y $26 \%$ en las mismas altitudes. Las afectaciones en árboles fueron leves, los daños en los frutos fueron más frecuentes por encima de los $300 \mathrm{msnm}$, con $20 \%$. Las pérdidas económicas ocasionadas al productor fueron $C \$ 1,962.00$ en un ciclo productivo. Existen diferencias significativas en la afectación de producción con relación a los rangos altitudinales, ya que al menos un tratamiento es diferente de los otros dos, en alturas menores de 200 y de 200 a $300 \mathrm{msnm}$ la media es de 1.70 , y la diferencia en alturas mayores de $300 \mathrm{msnm}$ con 2.60 según la prueba mínima de diferencia significativa.

Palabras clave: Cacao; plagas; pérdidas económicas; rangos altitudinales.

\section{Summary}

This research was conducted in fifteen cacao productive units, distributed in five communities of Siuna; plague prevalence was evaluated as well as the extent of impact on fruit production, branches and flowers. For the collection of data, the technique used was by samples with systematic transects distributed in $12 \%$ by planting in altitudinal ranges under 200, 200 to 300 and above 300 (mosl). The information was collected through direct observation, and the economic losses were quantified.

\footnotetext{
[1] Ingeniero Agroforestal. Autor. Correo: lopezrizonorlan22 @yahoo.com

[2] Ingeniero Agroforestal. Autor. Correo: elder.floresoza@yahoo.es

[3] Máster en Docencia Universitaria. Coordinador del área de Recursos Naturales. Correo: jasmillı@yahoo.com

[4] Máster en Docencia Universitaria, Técnico IREMADES. Correo: montesiuna@yahoo.com
} 
The plague prevalence is $74 \%$ and the absence of $26 \%$ in heights exceeding 300 (mosl). The batch excels in the 15 plantations, with $50 \%$ at ranges under 200 mosl, $38 \%$ from 200 to 300 and $64 \%$ above 300 mosl, the squirrel with $34 \%, 39 \%$ and $26 \%$ in the same altitudes. The affectations on trees were slight; damages to the fruits were more frequent above 300 mosl, with $20 \%$. Economic losses caused to the producer were of $C \$ 1,962.00$ in a productive cycle.

There are significant differences in the production affectation in relation to the altitudinal ranges, since at least one treatment is different from the other two, in lower altitudes of 200 and from 200 to 300 mosl the average is 1.70 , and the difference in heights exceeding 300 mosl with 2.60 according to the test of significant minimum difference.

Keywords: Cacao; plagues; economic losses; efficacy; altitudinal ranges.

\section{Introducción}

El cacao es un árbol de uso múltiple, ya que sirve no sólo para producir semilla, sino también para reforestar y enriquecer los bosques, refugios de avifauna, enriquece el suelo y además al producirlo con las técnicas adecuadas se incrementa la economía de los productores que lo cultivan, pero como cualquier cultivo es atacado por plagas las cuales lo afectan desde la etapa de vivero hasta la producción.

En la actualidad, Nicaragua alcanza una producción anual de veintidós mil quintales, siendo el municipio de Waslala el mayor productor; en todo el país, se obtienen rendimientos promedio de 4 quintales por manzana en plantaciones adultas. La poca producción de cacao y el bajo rendimiento por manzana en las plantaciones actuales no permite satisfacer la demanda del mercado nacional e internacional (Navarro y Mendoza, P. 11).

El cacao nicaragüense ha logrado ubicarse en algunos mercados de cacao fino, especialmente en los europeos donde ha logrado excelentes precios por su calidad. Sin embargo, a nivel nacional el cacao tiene una gran demanda que ha provocado que tenga un mejor precio (Ibíd.).

Las plantaciones de cacao en el municipio de Siuna, se encuentran en un descenso productivo, producto del mal manejo que se les está dando, pese a que es un rubro que genera ingresos adicionales a las familias productoras, la afectación de plagas ha sido el principal problema en la baja producción por manzana, se está dando la proliferación de plagas (insectos, áfidos, hormigas, roedores y pájaros) que afectan las flores, frutos, y las ramas de los árboles. 
Con este estudio se determinaron los tipos de plagas y el grado de afectación, ya sea en las flores, frutos, hojas y ramas de Theobroma cacao en el Municipio de Siuna, específicamente en las comunidades El Carao, Come negro, Guayabo, Rosa Grande y Floripón, con muestreos mediante el uso de transectas sistemáticas, trazadas en un $12 \%$ de cada plantación muestreada.

\section{Materiales y métodos}

\section{Ubicación del estudio}

Se realizó en cinco comunidades ubicadas en el área rural del municipio de Siuna: El Carao, a $25 \mathrm{Km}$, el Come Negro a 28 km, el Guayabo a $52 \mathrm{Km}$, , Rosa Grande a unos $40 \mathrm{~km}$, vía Siuna -Waslala, y la comunidad El Floripón, a $15 \mathrm{Km}$, vía Siuna a Rosita. Estas comunidades presentan características afines entre 2000-2500 milímetros al año, con temperaturas promedio de $26^{\circ} \mathrm{C}$, alturas sobre el nivel del mar entre o-70o msnm y pendientes de $2 \mathrm{al} 45 \%$.

\section{Tipo de estudio}

Se encuentra bajo el paradigma cuantitativo observacional, porque se brinda la información según lo natural encontrado en el campo mediante una observación directa en los árboles de cacao, además se procesaran los datos mediante un análisis estadístico utilizando la fórmula del Chi cuadrado $\left(\mathrm{X}_{2}\right)$ para pruebas de independencia.

La prueba estadísticas de independencia a través del Chi cuadrado para determinar si existe relación o independencia entre las variables de zonas y presencia de plagas, zonas y niveles de daños, de tal manera que permita poder ver los niveles de significancia entre las zonas y la presencia y afectación de las plagas en cacaotales.

\section{Universo}

Está comprendido por las plantaciones de cacao en las comunidades de El Carao, Come negro, Guayabo, Rosa Grande y Floripón.

\section{Muestra}

La muestra estuvo conformada por tres fincas de muestreos en cada una de las comunidades, las cuales conforman un total de 15 fincas muestreadas, en donde se tomarán en cuenta los rangos altitudinales: menor a $200 \mathrm{msnm}$; 200-300 msnm y mayor a $300 \mathrm{msnm}$. 


\section{Unidad de análisis}

Los árboles dentro del rango de las líneas transectas, las cuales se distribuyeron en un rango del 12\% de cada plantación.

\section{Variables}

Tipos de plagas, grado de afectación, pérdidas económicas.

\section{Fuentes y obtención de datos}

a. Fuentes primarias: Información recabada con fichas técnicas bajo la observación directa de campo en las fincas las cuales fueron objeto de estudio.

b. Fuentes secundarias: Literatura física, revistas e información que nos ofrece Internet.

\section{Técnicas e Instrumentos}

Técnica de registro estructurado de observación.

A través de un formato estructurado se levantó información sobre las principales plagas, parte del árbol afectada y nivel de afectación por las mismas.

\section{Resultados y discusión}

\section{Prevalencia de plagas en cacaotales}

La prevalencia de plagas (ausencia y presencia) en cacaotales, en tres estratos altitudinales de acuerdo al número de árboles afectados y al tipo de plagas, dio los siguientes resultados.

\section{Presencia y ausencia de plagas en cacaotales}

La presencia de plagas con relación a los rangos altitudinales fue: a más de $300 \mathrm{msnm}$ $74 \%$. En rangos de $200-300 \mathrm{msnm}, 47 \%$ y en altitudes menores de $200 \mathrm{msnm}, 58 \%$. Con relación a la ausencia en rangos mayores de $300 \mathrm{msnm}$ fue de $26 \%$, de 200-300 msnm $53 \%$ y menores de $200 \mathrm{msnm} 42 \%$.

Estos resultados se relacionan con lo planteado por Navarro, M, y Mendoza, I (Estudio de interacciones de hábitat de plagas según el nivel de vegetación); quienes plantean que a niveles más altos en donde la vegetación boscosa se mantiene en interacción con diferentes sistemas de cultivos anuales y perennes (cacao, café, musáceas, 
mango, papaya, etc., las plagas pueden proliferar de gran manera y crear un hábitat estable, causando un daño porcentual considerable en diferentes plantaciones de cultivos, provocando daños desde la etapa larvaria hasta la etapa adulta. Estos investigadores plantean que entre las plagas que más se encuentran son los minadores de frutos, tortuguillas, gusanos defoliadores, chinches, roedores etc., Estos datos se relacionan con nuestra investigación ya que este tipo de insectos son los que más afectan las plantas de cacao desde la etapa de floración hasta la maduración de los frutos. Cuadro No. 1.

Cuadro No. 1. Nivel de incidencia de plagas en el cacao con relación a los rangos altitudinales, Siuna, 2011

\begin{tabular}{|l|c|c|}
\hline \multirow{2}{*}{ Niveles altitudinales } & \multicolumn{2}{|c|}{ Incidencia de plagas } \\
\cline { 2 - 3 } & Presencia de plagas & Ausencia de plagas \\
\hline$-200 \mathrm{msnm}$ & $58 \%$ & $42 \%$ \\
\hline $200-300 \mathrm{msnm}$ & $47 \%$ & $53 \%$ \\
\hline$+300 \mathrm{msnm}$ & $74 \%$ & $26 \%$ \\
\hline
\end{tabular}

\section{Tipo de Plagas en cacaotales}

Los tipos de plagas que más se encuentran en plantaciones de cacao en los diferentes rangos altitudinales fueron: Mistletoes, Tanda (Phoradendrom sp) con 64\% en alturas mayores de $300 \mathrm{msnm} 38 \%$ en alturas de $200-300 \mathrm{msnm}$ y $50 \%$ en alturas menores de $200 \mathrm{msnm}$, estas infestaciones son causadas por los altos porcentajes de sombreamiento en las plantaciones y por precipitaciones considerables, específicamente en épocas de invierno, estos datos coinciden con lo que plantean Salgado M, Ibarra G, que la tanda es una de las plagas vegetales que más afecta el desarrollo y producción en plantaciones donde no se aplica un buen manejo agronómico. Cuadro No. 2.

Afectación por Ardillas: 26\% en alturas mayores de $300 \mathrm{msnm}$, 39\% en alturas de 200-300 msnm y 34\% en alturas menores de $200 \mathrm{msnm}$. Otras plagas encontradas son gusanos barrenadores del fruto, carpinteros, y comején los cuales se encuentran en bajos porcentajes no mayor a los $8 \%$ correspondientes en las diferentes zonas.

Considerando los tipos de plagas en los diferentes rangos altitudinales, podemos afirmar que a mayores alturas la tanda es la que más afecta, pero en alturas de 200-300 msnm la Ardilla causa daños severos en la producción ya que afectan directamente los frutos de cacao, Salgado M, Ibarra G, plantean que en el momento de la cosecha la plaga que más ataca es la ardilla, debido a que se alimenta del mucílago. Estos resultados también coinciden con (Marín y Gaitán; 2011) quienes plantean que en el municipio de Siuna las plagas que más afectan la producción de cacao son ardillas, tanda y gusanos. 
Cuadro No. 2. Porcentajes y tipos de plagas en cacaotales según rangos altitudinales

\begin{tabular}{|l|l|l|l|l|l|l|l|}
\hline \multirow{2}{*}{$\begin{array}{c}\text { Rangos } \\
\text { altitudinales }\end{array}$} & \multicolumn{7}{|c|}{ Plagas } \\
\cline { 2 - 9 } & Tandas & Ardilla & Chinche & Hormiga & Comején & Pájaro & Gusanos \\
\hline+300 & 64 & 26 & & & 8 & & \\
\hline $200-300$ & 38 & 39 & 1 & 3 & 3 & & 7 \\
\hline-200 & 50 & 34 & & 4 & 5 & 6 & 1 \\
\hline
\end{tabular}

\section{Nivel de afectación de las plagas en el cacao}

\section{Nivel de afectación en frutos}

La afectación de los frutos en las plantaciones de cacao varía según el rango altitudinal, en rangos menores a $200 \mathrm{msnm}, \mathbf{1 6 . 9 4 \%}$, de 200 a $300 \mathrm{msnm}, 20.71 \%$ y mayores de 300 msnm. 19.32\%. En alturas menores de 200 msnm el daño leve representa un 63\%, el moderado $31 \%$ y el grave $6 \%$. De $200-300$ msnm, daño leve $51 \%$ moderado $35 \%$ y grave $14 \%$, en los niveles mayores de 300 msnm con $20 \%$ de daño grave. Balarezo, realizó un estudio sobre la recolección de frutos de cacao en épocas de verano, y determinó que de cada 35 mazorcas aprovechables, 5 mazorcas fueron afectadas por Ardilla y carpintero, es decir, casi el $15 \%$ de los frutos fueron afectados, esta información coincide con nuestro estudio ya que se presentan daños de $15 \%$ y porcentajes más altos sobre frutos afectados. Cuadro No. 3.

Cuadro No. 3. Afectación por plagas en frutos de cacao con relación a rangos altitudinales

\begin{tabular}{|c|c|c|c|c|c|c|c|c|c|}
\hline $\begin{array}{c}\text { Rangos } \\
\text { altitudinales }\end{array}$ & \multicolumn{3}{|c|}{ Menos de 200 } & \multicolumn{3}{|c|}{$200-300$} & \multicolumn{3}{c|}{ Más de 300} \\
\hline $\begin{array}{c}\text { Nivel de } \\
\text { afectación }\end{array}$ & L & M & G & L & M & G & L & M & G \\
\cline { 2 - 11 } & 63 & 31 & 6 & 51 & 35 & 14 & 40 & 40 & 20 \\
\hline
\end{tabular}

$\mathrm{L}=$ leve, $\mathrm{M}=$ moderado, $\mathrm{G}=$ Grave.

\section{Comparación entre el porcentaje de afectación del fruto y los rangos altitudinales}

Basado en la prueba de Friedman, existen diferencias significativas, entre los niveles altitudinales con relación al porcentaje de afectación de los frutos en los cacaotales, siendo en nivel de mayor altura (más de $300 \mathrm{msnm}$ ), el que presenta los mayores porcentajes de afectación. 
Cuadro No. 4. Prueba estadística de Friedman

\begin{tabular}{|l|l|l|l|l|l|l|}
\hline \multicolumn{1}{|c|}{ Menor a 200 } & C200-300 & Mayor a 300 & \multicolumn{1}{|c|}{ T2 } & \multicolumn{1}{|c|}{ p } & & \\
\hline 1.70 & 1.70 & 2.60 & 67.69 & +0.0001 & & \\
\hline Mínima diferencia significativa entre suma de rangos (0.272) & \multicolumn{1}{|l|}{} \\
\hline Tratamiento & $\begin{array}{l}\text { Suma } \\
\text { ranks }\end{array}$ & Media ranks & $\mathrm{n}$ & & & \\
\hline C 200-300 & 80.00 & 1.70 & 47 & $\mathrm{~A}$ & & \\
\hline Menor a 200 & 80.00 & 1.70 & 47 & $\mathrm{~A}$ & $\mathrm{~B}$ & \\
\hline Mayor a 300 & 122.00 & 2.60 & 47 & & & $\mathrm{C}$ \\
\hline
\end{tabular}

Letras distintas indican diferencias significativas $(p+=0.050)$

Según la prueba estadística de Friedman existen diferencias significativas en uno de los tratamientos (mayor a $300 \mathrm{msnm}$ ) con relación a los demás tratamientos, donde hay una correlación positiva, según el porcentaje de afectación de frutos de cacao por plagas con relación a los niveles altitudinales, lo que nos indica que a mayor altura sobre el nivel del mar, mayor es el daño de los frutos en las plantaciones de cacao.

\section{Nivel de afectación por plagas en ramas productivas}

En rangos mayores de $300 \mathrm{msnm}$, el nivel es grave con $75 \%$, mientras que en los niveles menores de 200 hasta $300 \mathrm{msnm}$ no existe mucha diferencia con relación a los porcentajes de afectación. Cuadro No. 5.

Dentro del 75\% de afectación la cual es considerada grave se observaron daños severos por la presencia de las plagas comején y tanda los cuales inciden en un alto porcentaje en la afectación y daño de ramas; causando daños que provocan la muerte del árbol y en sí la baja producción de cacao. Esto se debe al mal manejo de la plantación. En alturas menores de $300 \mathrm{msnm}$ los daños no son significativos. Salgado M. Ibarra G., explican que en la mayoría de las plantaciones de cacao el comején, la hormiguilla y la polilla, son capaces de destruir todo el desarrollo vegetativo de cualquier especie arbórea con el fin de construir un hábitat estable dentro del marco de la vegetación, estos datos coinciden con los datos obtenidos en nuestra investigación, ya que las plagas persisten a mayores porcentajes de sombra en las plantaciones.

Cuadro No. 5. Porcentajes de afectación en ramas productivas con relación a los rangos altitudinales

\begin{tabular}{|c|c|c|c|c|c|c|c|c|c|}
\hline $\begin{array}{c}\text { Rangos } \\
\text { altitudinales }\end{array}$ & \multicolumn{3}{|c|}{ Menos de 200 } & \multicolumn{3}{c|}{$200-300$} & \multicolumn{3}{c|}{ Más de 300} \\
\hline $\begin{array}{c}\text { Nivel de } \\
\text { afectación }\end{array}$ & L & M & G & L & M & G & L & M & G \\
\cline { 2 - 11 } & 30 & 30 & 40 & 28 & 36 & 36 & 3 & 22 & 75 \\
\hline
\end{tabular}

(L: Leve; M: Moderado; G: Grave). 


\section{Nivel de afectación por plagas en flores}

La afectación en los rangos altitudinales menores de $200 \mathrm{msnm}$ fue leve, con 100\%, de $200-300$ msnm fue leve con 93\% y moderada con $7 \%$ y en rangos mayores de 300 msnm no se identificaron afectaciones. Los insectos causantes del daño a los cojines florales en su mayoría son chinches y zompopos. Carrasco L, Benítez C., plantean que la mayoría de los daños causados en las flores son ocasionados por las hormigas, zompopos, chinches y en menos porcentaje las aves, estos planteamientos coinciden con nuestra investigación ya que este tipo de insectos son los agentes causales de daño en las flores.

Cuadro No. 6. Porcentajes de afectación en flores con relación a los rangos altitudinales

\begin{tabular}{|c|c|c|c|c|c|c|c|c|c|}
\hline $\begin{array}{c}\text { Rangos } \\
\text { altitudinales }\end{array}$ & \multicolumn{2}{|c|}{ Menos de 200} & \multicolumn{3}{c|}{$200-\mathbf{3 0 0}$} & \multicolumn{3}{c|}{ Más de 300} \\
\hline $\begin{array}{c}\text { Nivel de } \\
\text { afectación }\end{array}$ & L & M & G & L & M & G & L & M & G \\
\cline { 2 - 10 }$y$ & 100 & & & 93 & 7 & & & & \\
\hline
\end{tabular}

\section{Pérdidas económicas causadas por plagas en plantaciones de cacao}

Las pérdidas económicas causadas por las plagas fue de 2310 frutos por manzana con promedios de 440 frutos afectados, esta cantidad representa el $19 \%$ de la cosecha perdida por manzana, en cacao seco representa 0.42 quintales (42 libras), el precio por libra se cotiza en $C \$ 23.00$, el productor pierde aproximadamente $C \$ 981$ córdobas por manzana en una corta. Las cortas picos se dan dos veces al año, esto significa que el productor pierde 1962 córdobas en el ciclo productivo.

A nivel municipal se considera que existen alrededor de 600 manzanas productivas, si tomamos en cuenta el ataque de plagas se perderían 252 quintales de cacao, valorado en lo acopios locales a un precio variable de $C \$ 2,300.00$, equivale a $C \$ 579,600.00$ córdobas perdidos a causa de la afectación por plagas.

Cuadro No. 7. Pérdidas económicas causadas por plagas en plantaciones de cacao

\begin{tabular}{|l|l|l|l|l|l|}
\hline $\begin{array}{c}\text { Rangos } \\
\text { altitudinales }\end{array}$ & $\begin{array}{c}\text { Frutos } \\
\text { cosechados/ } \\
\mathbf{m z}\end{array}$ & $\begin{array}{c}\text { Frutos } \\
\text { afectados }\end{array}$ & $\begin{array}{c}\text { \% } \\
\text { pérdida }\end{array}$ & $\mathbf{Q q ~ / m z}$ & $\begin{array}{c}\text { Pérdidas } \\
\text { económicas } \\
\mathbf{C \$}\end{array}$ \\
\hline+300 & 2088 & 335 & 16.94 & 0.30 & 690 \\
\hline $200-300$ & 3631 & 752 & 20.71 & 0.75 & 1725 \\
\hline-200 & 1211 & 234 & 19.32 & 0.23 & 529 \\
\hline Promedios & 2,310 & 440 & 19 & 0.42 & 981 \\
\hline
\end{tabular}




\section{Lista de referencia}

Balarezo Daniel. Revista Informativa Agropecuaria., Ed. 158, p: 11. Disponible en www. google.com/revistasinformativas.

Carrasco L, Benítez C. Manejo de plantaciones de Theobroma cacao. Disponible en: www. google.com/Theobromacacaomanejo.

Enríquez G. Plagas y enfermedades del cacao. Disponible en http://www.infoagro.go.cr/ agricola/tecnologia/cacao/pyenfermedades.htmGustavo Enríquez.

Escobedo, A. (2009). Cadena de Valor del cacao en Costa Rica. 24p.

Disponible en: http://orton.catie.ac.cr/repdoc/A7712E/A7712E.PDF.

Flores Mendoza, J. (2000). Insectos asociados a las variedades de cacao cultivadas en el CACABO Municipio de bonanza en la época lluviosa del año 2000.

Marín A, y Gaitán M. (2011). Árboles Potenciales de (Theobroma cacao) en el Municipio de Siuna 2009-2010. 64 p. URACCAN.

Morales y Vargas. (1982). Estudios de la realización de la densidad de las poblaciones de especies de insectos en cacao. El cacaotero.

Navarro, M y Mendoza, I. (2006). Cultivo del cacao en Sistemas Agroforestales. Guía técnica para promotores. Disponible en:http://www.iica.int.ni/Estudios_PDF/ Guia_Cacao_Para_Promotores.pdf.

Salgado M, Ibarra, G. (Prácticas de Agricultura). Para el Manejo del cultivo de Theobroma cacao. CATIE, Costa Rica, 2007. 55p.Disponible en www.google. com/practicasdeagricultura/cacao.

Saunders, J y Enríquez, G. (1989). Manejo integrado de plagas insectiles. 